\title{
Review
}

Psychopathology

Psychopathology 2019;52:153-160

DOI: 10.1159/000500294
Received: March 29, 2018

Accepted after revision: April 11, 2019

Published online: June 6, 2019

\section{Arguments for a Phenomenologically Informed Clinical Approach to Autism Spectrum Disorder}

\author{
Maria Nilsson ${ }^{a, b}$ Peter Handest $^{c}$ Lena Nylander ${ }^{d, e} \quad$ Lennart Pedersen $^{f}$ \\ Jessica Carlsson ${ }^{\mathrm{a}, \mathrm{b}}$ Sidse Arnfred ${ }^{\mathrm{b}, \mathrm{g}}$

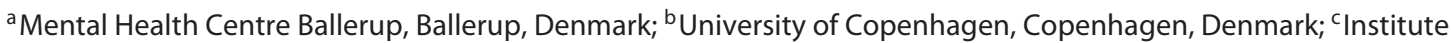 \\ for Mental Health, Herlev, Denmark; ${ }^{d}$ Department of Clinical Sciences/Psychiatry, Lund University, Lund, Sweden; \\ ${ }^{e}$ Gillberg Neuropsychiatry Centre, University of Gothenburg, Gothenburg, Sweden; ${ }^{f}$ Center for Autism, Herlev, \\ Denmark; ${ }^{9}$ Mental Health Services - Region Zealand, Slagelse, Denmark
}

\section{Keywords \\ Phenomenology · Psychopathology · Autism spectrum disorder · First-person experience}

\begin{abstract}
Objective: The aim of this paper is to provide arguments for a phenomenologically informed clinical approach to autism spectrum disorder (ASD), including a plea for clinical attention to the self in ASD. Methods: Central concepts of continental phenomenology, phenomenological psychopathology, and the phenomenological interview are presented, with an emphasis on the potential unifying qualities of an approach which includes the exploration of subjective and intersubjective experience. These phenomenological concepts and methods are contrasted with the current conceptualization of ASD, where the first-person perspective is not in focus. Results: Contemporary phenomenological papers on ASD address key concepts like intersubjectivity, intercorporeality, and intentionality. However, insights from this theoretical field have not been followed up in clinical research and practice. Consequently, there is (to our knowledge) still a lack of phenomenologically informed clinical explorations of experience of self, others, and the world in ASD.
\end{abstract}

Conclusion: A phenomenologically informed focus on the form and structure of subjective experience, including a focus on self-experience in ASD, can lead to new and important insights in relation to clinical differentiation between ASD and schizophrenia spectrum disorder.

(c) 2019 S. Karger AG, Basel

\section{Introduction}

Phenomenologists have long argued that a phenomenological frame of reference would make crucial contributions to our insight into autism spectrum disorder (ASD) [1-3]. The main argument for a phenomenological approach is that phenomenology offers concepts and methods for the study of form and structure of subjective experience [4], a perspective that has not been in focus in mainstream ASD research. The exploration of subjective experience provides a unique possibility to enter the realm of how people with ASD experience themselves, others, and the world, a question that has puzzled scientists and family members alike since the emergence of ASD as a diagnostic concept. As we shall argue below, insight into the first-person mode of experience is also of

\section{KARGER}

(c) 2019 S. Karger AG, Basel 
central value to unify the many angles of the current mainstream accounts of ASD.

Several theoretical accounts of the mode of subjective experience in ASD have been presented by phenomenologists like Raffman [2], Gallagher [5], Zahavi [6], and Fuchs [7]. Nevertheless, the application of this work in clinical ASD research and practice, with personal contact to patients diagnosed with ASD, is still lacking. We will therefore present arguments in favor of a clinical phenomenological approach. To provide a basis for these arguments, we briefly draw up the historical and current status of clinical ASD research, provide an overview of the main concepts of phenomenology and phenomenological psychopathology and, finally, present the main concepts of the existing phenomenological work on ASD. Hereafter, a basis for our methodological considerations is given in a section on the main concepts of a phenomenological semi-structured interview. In the light of these accounts, we argue for the clinical benefits of a deeper insight into the mode of experience in ASD, with implications for both clinical research and differential diagnostic practices. We also discuss whether such an approach is feasible in a clinical ASD population as well as give an initial bid as to how a phenomenologically oriented interview exploring subjective experience in ASD could be approached.

\section{Historical and Current Status of Mainstream ASD Research}

ASD was first described by Kanner [8] and Asperger [9] in the 1940s. Both authors drew on the already-known Bleulerian [10] concepts from schizophrenia spectrum psychopathology. In accordance with these concepts, they both (with some variation) used the term "autism" to emphasize a deficient contact with others/the world as being a central feature of ASD.

Kanner [8] stressed that his autistic patients showed a fundamental inability to relate to others. He described this as a trait that had been present from the very beginning of their lives. To prove his point, he cited parents stating that their children were "self-sufficient," "like in a shell," and "perfectly oblivious to everything about him."

Similarly, Asperger [9] concluded that "the autist is only himself" (cf. the Greek word "autos") as opposed to a part of our shared world. He also claimed that "the essential abnormality in autism is a disturbance of the lively relationship with the whole environment" (p. 74), and that "If somebody can only experience in an original way, and if he can only be "his own self rather than feel himself to be an integral part of the world /.../ then he is unable to learn" (p. 63) [11]. What is important to note here is the description of a certain lack of reciprocity with the world (and not the somewhat arbitrary conclusions on learning ability), and the fact that both Kanner's and Asperger's accounts somehow roam the outskirts of a description of children's relation to the world. However, in accordance with the main clinical and scientific approach at the time, both authors described ASD from the point of view of the observer (i.e., the third-person perspective) [12].

In 1962, Bosch [13] published rare clinical phenomenological examinations of the mode of existence (the being-in-the world) in ASD. In his book, Infantile Autism: A Clinical and Phenomenological-Anthropological Investigation Taking Language as the Guide, he drew on phenomenological work by Binswanger [57] and Minkowski $[58,59]$, and described inadequate concepts related to acting (engaging) and having (possessing) in ASD. Similar to Kanner's and Asperger's considerations, he also concluded that a child with ASD "...fails to find a way into the realm of encounter, to the constitution of an own and common world" (p. 115). He described this as a distance or "glass wall" in the encounter, which he claimed was due to a unidimensional, goal-directed relation to the world in ASD. However, though he built on phenomenological concepts, Bosch's descriptions were also observational, i.e., from a third-person rather than a first-person perspective.

A multitude of clinical advances have subsequently been made towards an understanding of the mind in ASD. Most of these have been within the realm of mindtheory $[14,15]$, the central coherence-theory [16], or concerning deficits in executive function [17]. Common for these classical psychological theories is a focus within the frameworks of cognitive neuroscience and neuropsychology $[14,16]$, neither of which have the first-person perspective in focus. Instead, the concepts and methods of both have led to the current conceptualization of ASD which is characterized by descriptions of (isolated) cognitive mechanisms and behavioral features.

It has, however, been argued that the lack of focus on the first-person experiential dimension in most of the historical and current ASD research results in a lack of precision regarding current theoretical claims about "what the problem is" in ASD, since a crucial level of explanation is missing (i.e., the first-person experiential level) $[1,18]$. 


\section{Phenomenology and Phenomenological Psychopathology}

In contrast, the phenomenological and phenomenological psychopathological traditions specifically focus on a detailed exploration of the first-person perspective [4]. Below, the central concepts of these approaches are outlined.

\section{Phenomenology}

Phenomenology is a major philosophical tradition, initiated by Husserl [19]. As a scientific discipline, it is concerned with the basic structures of subjective and intersubjective experience. What is sought to be explored are the essential features of the first-person perspective, sometimes referred to as the "what it is like" of conscious experience [20].

When addressing the "what it is like-ness" of experience from a phenomenological (philosophical) stance, it is important to first differentiate between descriptions of how we think (mode/form), what we think (content), and why we think like we do (insight/understanding). This distinction is important, as the literature contains several different definitions of the term phenomenology that target different aspects of subjective experience. The definition of phenomenology used in this paper is in the tradition that is specifically devoted to exploration of how we think, i.e., the prereflective form and structure of experience [1]. This contrasts with the broader use of the term, i.e., phenomenology stands for either first-person descriptions in general (with a focus on content and/or introspection) or (as in psychiatry) as a synonym for the description of regularly occurring symptoms $[1,21]$.

According to phenomenology, an essential structure of subjectivity is the notion of prereflective self-awareness [22]. This basic form of self-awareness is to be understood as the first-person "givenness" of experience, i.e., the fact that you know that it is your experience, without having to think about it. This first-person givenness is a formal quality of experience, necessary for any experience to be understood as one's own (i.e., to be experienced from a first-person perspective) $[22,23]$. Other examples of essential and mutually intertwined formal qualities of human consciousness are: the notion that lived time is prereflectively experienced as a continuum, i.e., with past, present, and future dimensions (temporality) [22, 24]; and that consciousness is always implicitly directed against something, i.e., with the world as a reference (intentionality) [20].

Phenomenological Psychopathology and ASD
During the development of phenomenological concepts over a century, it was further emphasized that subjective experience is embodied and contextually embedded. Embodiment stands for the notion that, in our general way of experiencing, we experience with our mind and body in unison, i.e., the self is not experienced as being isolated from its surroundings, but rather as an embodied self [25]. Likewise, our being-with-others is with our mind and body in unison (intercorporeality) [7]. We are also prereflectively attuned to others (intersubjectivity) [20] and the context in which we find ourselves. Building on these concepts, the phenomenological tradition offers a coherent conceptual (and methodological) framework for the exploration and description of human subjectivity $[1,20]$. It has therefore been argued that this tradition is of specific scientific value for psychopathology and psychiatry, where subjective experience is altered in one way or another $[4,26]$.

\section{Phenomenological Psychopathology}

Phenomenological psychopathology draws on these concepts to describe the variations in subjective and intersubjective experience that are associated with mental disorders [20,27]. These variations in patients' prereflective mode of experience (their being-in-the world) are accounted for in both classical and contemporary phenomenologically informed literature as being close to the core of what constitutes the respective disorders $[4,26]$. The tradition of phenomenological psychopathology was founded by Jaspers [28]. As in the philosophical phenomenological tradition, the focus is on the form and structure of subjective experience, with the aim of grasping experiential variations specific to the disorder in question.

In this section, we use selected parts of the extensive phenomenological psychopathological tradition, in relation to schizophrenia spectrum disorder, to illustrate which insights could potentially be gained with this approach. In phenomenological terms, schizophrenia spectrum disorder is described as a disorder of basic selfawareness (ipseity), with a pervasive and persistent shift of the first-person perspective. This shift consists of a diminished sense of prereflective self-presence and an exaggerated self-consciousness (hyperreflectivity). Therefore, instead of experiencing thoughts, feelings, and sensations with an intrinsic first-person givenness (mineness), subjective experience is as if from a "third-person perspective" $[29,30]$. Other examples of variations in subjective experience encompass a loss of vital contact with reality, i.e., a lack of prereflective awareness. Minkowski de- 
scribes this as the "trouble générateur" or "generating disorder" in schizophrenia; Blankenburg's common sense crisis stands for a lack of an implicit understanding of everyday life [31, 32]; and Conrad's concept of apophany represents a specific experience of "being-stricken-by" delusional meaning, characteristic of the early phases of schizophrenia [33]. For a more comprehensive account on schizophrenia spectrum psychopathology, in the phenomenological sense, please refer to Gallagher and Zahavi [20] and Broome et al. [34].

Central to the argument in this paper is that these concepts are all described as essential features of how the mere structure of experience of self, others, and the world (consciousness) is altered in schizophrenia. This alteration of subjective experience is often present long before the development of classical schizophrenia spectrum symptomatology, and it is described as serving essentially as a unifying matrix, or gestalt, from which the classical symptoms and signs arise $[29,35,36]$.

\section{Contemporary Phenomenological Accounts of ASD Psychopathology}

Similar to the phenomenologically informed approach to schizophrenia spectrum disorder, there are several theoretical (nonclinical) accounts of how phenomenological concepts can inform our understanding of subjective experience in ASD. A large proportion of these accounts can be divided into work on (1) intersubjectivity and (2) the self/self-awareness.

\section{Intersubjectivity in ASD}

Many of the phenomenological accounts of ASD address the lack of attuned relation to others and the world, touched upon already by both Kanner [8] and Asperger [9]. Phenomenologists describe this lack of relationality as an essential structure of conscious experience in ASD that includes disturbances of intentionality, intersubjectivity, and intercorporeality. However, both Fuchs [7] and Gallagher [5] argue that, in order to fully describe the essence of ASD, a leveled approach to intersubjectivity is also needed. With reference to the work of Trevarthen $[60,61]$, Gallagher [37] argues that intersubjective interaction consists of both a primary and a secondary intersubjectivity. Primary intersubjectivity allows infants to imitate facial gestures and attune to smiles and familiar voices. In phenomenological terms, this is to prereflectively engage with others [38]. Secondary intersubjectivity, on the other hand, additionally encompass a prereflective understand- ing of other people's actions as intentional and part of a contextual whole, corresponding somewhat to the neurocognitive concept of central coherence [16]. In addition, Fuchs [7] defines a third level, tertiary intersubjectivity, which implies a self-other meta-perspective, corresponding to the theory of mind/mentalizing concept [15]. Fuchs [7] and Fuchs and de Jaegher [39] also emphasize the bodily features of primary intersubjectivity, where a common meaning is created through embodied interaction (intercorporeality). The basic concepts of the theoretical phenomenological account of ASD psychopathology are summarized in the following citations from Fuchs [7]: "From a phenomenological approach, autism should rather be conceived as a disorder of primary or embodied intersubjectivity" (p. 196), i.e., a lack of pre-reflective and embodied attunement with others, and that "disturbances arise on the higher level of intersubjectivity too, but they are based on the primary disturbances of embodied interaction and attunement" (p. 193). What is indicated by these citations, and central to the argumentation of this paper, is that the levelled approach to intersubjectivity captures a unifying layer, "below" the concepts described in the neurocognitive approach.

In a recent paper, Grohmann [40] further elaborates on the intentional body in ASD. With reference to data on sensimotor function in ASD and in line with the embodied accounts of intersubjectivity by Fuchs and de Jaegher [39], he argues that the body-world relationship is disentangled in ASD. The general, more open reference to the world is replaced by an exaggerated focus on sole tasks, with a narrowed bodily intentionality. He concludes that this lack of a larger perceptual horizon leads to the lack of a coherent sense of intentional meaning of the world in ASD.

\section{Self/Self-Awareness in ASD}

On a slightly different note, Raffman [2] addresses the domain of self/self-awareness in ASD. In her 1999 paper, she approaches the self in much the same way as has been done in relation to schizophrenia spectrum disorder. In accordance with phenomenological concepts, she suggests a differentiation between 2 dimensions of self-awareness in ASD: a kind of self-awareness where introspection and theory of mind are involved, and another nonconceptual and thinner awareness of one's own conscious mental states. The thinner self-awareness does not involve a cognitive achievement, as no judgement or concept is applied, one is simply aware. She argues that it is only the thicker self-knowledge that is impaired in ASD, allowing for an unaffected first-person access to the thinner prereflective 
self-awareness. Zahavi and Parnas [1] further elaborate on Raffman's claim, with the argument that neurocognitive descriptions of subjective experience in ASD [41, 42] indicate the presence of a basic (prereflective) self-awareness. This approach has important qualities, both for the description/understanding of self-awareness in ASD and in relation to the at times difficult clinical differentiation from schizophrenia spectrum disorder $[43,44]$.

\section{The Clinical Phenomenological Semi-Structured Interview}

When addressing subjective experience, there are important methodological concerns to be noted. We therefore shift here to a methodological focus. The phenomenological tradition has developed suitable methods to adequately explore the form and structure of conscious experience, in the shape of a phenomenological interview [4]. The phenomenological interview aims to grasp essential (invariant) structures of the first-person experience (eidetic variation) [30, 45]. In a clinical diagnostic setting, this is best achieved with a semi-structured interview, i.e. an interview with predefined topics to cover, but with neither preformulated questions nor an ordered sequence in which the questions are to be asked [46]. Instead, the interview style is conversational and follows the patient's narrative. This approach allows for an in-depth exploration of the patient's subjective experiences, albeit with the focus maintained on topics relevant for diagnostic evaluation [46]. The interviewer must strive to explore the patient's experiences as they appear for the patient's "I," with a suspension (epoché) of their own beliefs and judgements (i.e., the interviewer's natural attitude). Furthermore, the interview must be conducted by way of reciprocity (the encounter between "yous"), as this allows for an approximate mutual exploration of the patient's firstperson perspective $[20,46]$. The patient's experiences are explored and described as details of a meaningful and coherent experiential whole, in accordance with Jaspers' [62] gestalt notion [47]. An identified gestalt can then be compared with other experience- and knowledge-based gestalts or diagnostic prototypes in the diagnostic process. It has been argued that this approach increases the precision of clinical diagnosis as complex, with essential nuances of the respective disorders nevertheless captured in a more coherent way $[21,48,49]$.

As noted above, these concepts and methods from phenomenology and phenomenological psychopathology have been reapplied in schizophrenia spectrum research

Phenomenological Psychopathology and ASD and clinical work over the last decades. A notable example of this reapplication is the Examination of Anomalous Self-Experience (EASE) scale described by Parnas et al. [30]. The EASE scale is a checklist for a semi-structured clinical phenomenological interview, based on persistent variations in self-experience (basic-self disturbances) described in relation to schizophrenia spectrum disorder [50]. Guided by phenomenological concepts, the scale targets experiential domains like cognition and stream of consciousness, self-awareness and presence, bodily experiences, demarcation/transitivism, and existential reorientation [30]. The scale has been used in clinical schizophrenia spectrum research for over a decade and has proven to have good psychometrical properties [50].

Zukauskas et al. [51] employ a similar method for the clinical exploration of subjective experience in ASD, with a focus on temporality and experience of time, in their 2009 paper. With the phenomenological perspective of Merleau-Ponty [63] as their point of reference, they approach temporality as a structure of existence and state that: "the purpose is to investigate and describe the experience of time related to everyday life and to the personal history of individuals with Asperger's syndrome" (p. 92). Based on their findings from clinical semi-structured phenomenological interviews, the authors conclude that present and future time was described in factual, repetitive, and stereotypical terms. By reciting daily routines using chronological and sequential references (minutes, hours, days, or sequences of routines) the participants delivered factual and literal descriptions of themselves and their personal life, exemplified by: "Interviewer: How could you explain "time" to me, please? M: It is a circle of hour. It is used to eat, have dinner and for everything you have to do" (p. 100). In contrast, the participants' past experiences were described with a more nuanced sense of temporality, where past and present (but not future) time was experienced as a continuum. True to the phenomenological method, Zukauskas et al. [51] claim to have been "bracketing theoretical presuppositions about the topics of lived time and Asperger's syndrome was fundamental in order to investigate the participant's experience of time and to consider him/her as being in the world" (p. 92).

\section{Discussion: A Phenomenologically Informed Clinical Approach to ASD}

We have briefly summarized the main concepts of historic and current ASD research, highlighting the recurrent lack of focus on the form and structure of subjective 
experience. Furthermore, we have outlined basic conceptual and methodological features of the phenomenologically informed approach, which is specifically devoted to the exploration of the form and structure of first-person experience. We will now address how clinical ASD research and diagnostics would benefit from the application of these concepts and methods.

From the early third-person descriptions of the lack of relation to others and the world in ASD, already one clearly gets the impression that this lack of relation, or inaccessibility, is something other than a self-chosen or deliberate isolation. However, if a patient's subjective experiences are not taken into account, how are we to know what the problem really is? How is this central lack of relation to the world experienced from the first-person perspective? Do people diagnosed with ASD experience themselves, others, and the world in a way that differs from the general way of experiencing? If so, what does (or doesn't) this mode of experience encompass? How does it differ from the form and structure of experience in other/clinically similar disorders, such as schizophrenia spectrum disorder? Phenomenology serves as a suitable framework to answer precisely these questions, all of which have evident value for a nuanced description and understanding of the mind in ASD.

The theoretical phenomenological accounts of beingin-the world in ASD as well as the clinical exploration by Zukauskas et al. [51] of temporality in ASD indicate that the phenomenological perspective has the potential to unify the current conceptualization of ASD. In effect, Zahavi and Parnas [1] argue that the addition of a subjective experiential domain would serve as a necessary link between existing neurocognitive data and subsequent claims as to what constitutes "the problem" in ASD. Along the same lines, Gallagher [5] argues that the key to understanding social difficulties in ASD may be found in the phenomenological concepts of embodied interaction and self-world relation. From a phenomenological perspective, ASD could be described as a disorder of intersubjectivity, intercorporeality, and intentionality. With further elaboration on these claims, and a refined understanding of the subjective and intersubjective experience in ASD, a coherent ASD gestalt may emerge. Based on the notion of prototypical diagnosis, this would enable further diagnostic precision $[48,52]$, with benefits for everyday clinical practice. With time, it might even be possible to describe some features of experience in a more explanatory manner, akin to the concept of a loss of "vital contact with reality" as being the "trouble générateur" (generating disorder) in schizophrenia [27].
However, despite these promising theoretical concepts and experimental indications, phenomenologically informed clinical research following up on these notions is, to our knowledge, still lacking. A reason for this could be that, at first glance, it may seem that this approach is unattainable in an ASD population. The traditional choice of a third-person approach in clinical ASD research and practice is logical for several reasons. Firstly, many studies and clinical diagnostic evaluations concern small children or people with severely affected verbal communication, which naturally hampers the feasibility of an in-depth clinical interview on subjective experience. Secondly, the conceptualization of ASD as a disorder signified by a lack of introspective awareness (the theory of mind/own mind) [15], could potentially lead to the belief that any endeavor to access the firstperson perspective would be futile, regardless of age and linguistic capacities. This notion is, however, contradicted by Hurlburt et al. [41], who reported a cognitive ability to think about inner experience in individuals with Asperger's syndrome, using an experience-sampling technique. These indications are further substantiated by Raffman [2] and Zahavi and Parnas [1] above, with the claims that neurocognitive data support the notion of an intact thinner or basic self-awareness in ASD, access to which does not require meta-cognition. $\mathrm{Zu}$ kauskas et al. [51] further confirm that individuals with Asperger's syndrome are able to participate in this kind of mutual exploration of subjective experience, and, furthermore, that this kind of approach yields fruitful descriptions of formal aspects of subjective experience in ASD.

All in all, these considerations speak for the phenomenologically informed clinical approach to ASD as being both conceptually rewarding and methodologically feasible.

\section{Future Tasks}

How, then, could a phenomenologically informed clinical exploration of subjective experience in ASD be approached? The significant proportion of phenomenological literature on intersubjectivity in ASD mirrors the intuitive value of an exploration of the intersubjective realm for gaining further insights into classical ASD symptomatology. We would like to argue for the additional importance of a focus on the self in ASD. This is in line with claims from both phenomenologists and neurocognitive researchers; both Elmose [53] and Zahavi [3] have called for clarification and refinement of the concepts of self and self-awareness in current ASD 
research. Zahavi [3] describes the self in ASD as a virtually undiscovered area, and points to the clarifying value of a future dimensional approach to the self in ASD research, consistent with phenomenological theory. $\mathrm{He}$ suggests a distinction of an experiential, interpersonal, and narrative dimension of the self, and goes on to define the task as a detailed exploration of these dimensions for the purpose of identifying possible selected impairments of the self in ASD, rather than the impairment of the self as a whole $[3,6]$. As briefly outlined above, this approach has proven successful in relation to further insights on what constitutes the essence of self-experience in schizophrenia spectrum disorder [31, 54]. In favor of the dimensional approach and informed by the advances made in schizophrenia spectrum research, we therefore argue that the use of the above-described EASE scale is a particularly interesting point of departure for the initial exploration of subjective experience in ASD. The EASE scale allows for a fine-grained clinical exploration of self-experience, including specific exploration of the indications of an intact basic self in ASD. This approach would not only shed a new and important light on the experiential self in ASD, it would also have possible differential diagnostic implications, linked to the above-described affected basic self in schizophrenia spectrum disorder.

In addition to a focus on the self, a clinical phenomenological approach to ASD would naturally have to be adapted and expanded according to the theoretical claims made by contemporary phenomenologists. Consequently, further clinical exploration of subjective experience in ASD would preferably encompass the domains of intersubjectivity, intercorporeality, and intentionality. For this purpose, it would prove useful to look to the recent proposal by Bizzari [55] to adopt an embodied approach to intersubjectivity in ASD, and also the recently released "EAWE" (Examination of Anomalous World-Experi- ence) [56]. The EAWE is developed from the concepts of the EASE scale and is a checklist for a semi-structured phenomenological interview on experiential domains like space and objects, time and events, other individuals, language, atmosphere, and existential orientation. In line with the theoretical phenomenological accounts of ASD, these are all relevant dimensions to explore in the endeavor to further substantiate the "being-in-the world" in ASD.

\section{Conclusion}

We have provided arguments for why a clinical phenomenological approach to ASD deserves more attention. It constitutes a conceptually coherent, highly valuable, and feasible approach, with the prospect of gaining new and unifying insights into both "what it is like" to be autistic and in relation to clinical differentiation between ASD and schizophrenia spectrum disorder.

\section{Acknowledgments}

Many thanks to Research Librarian Trine Lacoppidan Kæstel, Psychiatric Research Unit, Region Zealand Mental Health Services for the competent and committed assistance, and to the anonymous reviewers for their invaluable feedback on the paper's content and direction. Thanks also to Prof. Francesca Happé for valuable discussions with the first author (M.N.) on the wider topic of this paper.

\section{Funding Source}

The work was carried out with funding from the Mental Health Services in the Capital Region of Denmark, The Capital Region of Denmark and Sofiefonden. Handest and Nilsson has received honoraria from Lundbeck and Otsuka for teaching psychopathology.

\section{References}

1 Zahavi D, Parnas J. Conceptual problems in infantile autism research: why cognitive science needs phenomenology. J Conscious Stud. 2003;10(9-10):9-10.

2 Raffman D. What Autism May Tell Us about Self-Awareness: A Commentary on Frith and Happé. Mind Lang. 1999;14(1):23-31.

3 Zahavi D. Complexities of self. Autism. 2010 Sep;14(5):547-51.

4 Fuchs T. Subjectivity and intersubjectivity in psychiatric diagnosis. Psychopathology. 2010;43(4):268-74.

Phenomenological Psychopathology and ASD
5 Gallagher S. Intersubjectivity and Psychopathology. In: Fulford KWM, Davies M, Gipps RGT, Graham G, SadlerJZ, StranghelliniG, etal, editors. The Oxford Handbook of Philosophy and Psychiatry. Oxford: University Press; 2013.

6 Zahavi D. Self \& Other: exploring subjectivity, empathy, and shame. Oxford: University Press; 2014

7 Fuchs T. Pathologies of intersubjectivity in autism and schizophrenia. J Conscious Stud. 2015;22:191-214.

8 Kanner L. Autistic disturbances of affective contact. Nerv Child. 1943;2:217-50.
9 Asperger H. Die 'Autistischen Psychopathen' im Kindesalter. Arch Psychiatr Nervenkr. 1944;117:76-136.

10 Bleuler E. Dementia praecox oder Gruppe der Schizophrenien. In: Handbuch der Psychiatrie. Leipzig: Deuticke; 1911.

11 Frith U. Autism and Asperger syndrome. Cambridge: University Press; 1991.

12 Berrios GE. The History of Mental Symptoms: Descriptive Psychopathology since the Nineteenth Century. Cambridge: University Press; 1996. 
13 Bosch G. Infantile Autism: A Clinical and Phenomenological-Anthropological Investigation Taking Language as the Guide. Berlin: Springer; 1970. German.

14 Happé F. Autism: An Introduction to Psychological Theory. New York: Routledge; 1994.

15 Baron-Cohen S, Leslie AM, Frith U. Does the autistic child have a "theory of mind"? Cognition. 1985 Oct;21(1):37-46.

16 Happé F, Frith U. The weak coherence account: detail-focused cognitive style in autism spectrum disorders. J Autism Dev Disord. 2006 Jan;36(1):5-25.

17 Ozonoff S, Pennington BF, Rogers SJ. Executive function deficits in high-functioning autistic individuals: relationship to theory of mind. J Child Psychol Psychiatry. 1991 Nov; 32(7):1081-105.

18 Hobson RP. On acquiring knowledge about people and the capacity to pretend: response to Leslie (1987). Psychol Rev. 1990 Jan;97(1): $114-21$.

19 Husserl E. Logical Investigations. Volume 1. New York: Routledge; 2001.

20 Gallagher S, Zahavi D. The phenomenological mind, an introduction to philosophy of mind and cognitive science. New York: Routledge; 2008.

21 Andreasen NC. DSM and the death of phenomenology in america: an example of unintended consequences. Schizophr Bull. 2007 Jan;33(1):108-12.

22 Zahavi D. Subjectivity and Selfhood: Investigating the First-Person Perspective. Cambridge (MA): MIT Press; 2008.

23 Zahavi D. Beyond Empathy: Phenomenological Approaches to Intersubjectivity. J Conscious Stud. 2001;8(5-7):151-67.

24 Fuchs T. Temporality and psychopathology. Phenomenol Cogn Sci. 2013;12(1):75-104.

25 Fuchs T, Schlimme J. Embodiment and psychopathology: a phenomenological perspective. Curr Opin Psychiatry. 2009;22(6):570-5

26 Owen G, Harland R. Theme issue on phenomenology and psychiatry for the 21st century. Taking phenomenology seriously. Schizophr Bull. 2007 Jan;33(1):105-7.

27 Sass L. Explanation and description in phenomenological psychopathology. J Psychopathol. 2014;20(4):366-76.

28 Jaspers K. General Psychopathology. Volume 1. Baltimore (MD): Johns Hopkins University Press; 1997.

29 Sass LA, Parnas J. Schizophrenia, consciousness, and the self. Schizophr Bull. 2003;29(3): 427-44.

30 Parnas J, Møller P, Kircher T, Thalbitzer J, Jansson L, Handest P, et al. EASE: Examination of Anomalous Self-Experience. Psychopathology. 2005 Sep-Oct;38(5):236-58.

31 Stanghellini G, Ballerini $M$. What is it like to be a person with schizophrenia in the social world? A first-person perspective study on schizophrenic dissociality. Part 1: state of the art. Psychopathology. 2011;44(3):172-82.

32 Blankenburg W. First steps toward a 'psychopathology of common sense'. Philos Psychiatry Psychol. 2001;8:303-15.

33 Conrad K. Die Beginnende Schizophrenie. Stuttgart: Thieme; 1958. Danish translation. Handest P, Jansson L, et al. Den Begynende Skizofreni. Copenhagen: Munksgaard; 2006.

34 Broome MR, Harland R, Owen GS, Stringaris A, editors. The Maudsley Reader in Phenomenological Psychiatry. 2013.

35 Parnas J, Bovet P, Zahavi D. Schizophrenic autism: clinical phenomenology and pathogenetic implications. World Psychiatry. 2002 Oct;1(3):131-6.

36 Parnas J, Zandersen M. Self and schizophrenia: current status and diagnostic implications. World Psychiatry. 2018 Jun;17(2):220-1.

37 Gallagher S. Understanding Interpersonal Problems in Autism: Interaction Theory as An Alternative to Theory of Mind. Philos Psychiatry Psychol. 2004;11(3):199-217.

38 Gallagher S, Meltzoff A. The earliest sense of self and others: Merleau-Ponty and recent developmental studies. Philos Psychol. 1996. doi: 10.1080/09515089608573181.

39 Fuchs T, de Jaegher H. Enactive intersubjectivity: participatory sense-making and mutual incorporation. Phenomenol Cogn Sci. 2009; 8(4):465-86.

40 Grohmann TDA. A Phenomenological Account of Sensorimotor Difficulties in Autism: Intentionality, Movement, and Proprioception. Psychopathology. 2017;50:408-15.

41 Hurlburt RT, Happé F, Frith U. Sampling the form of inner experience in three adults with Asperger syndrome. Psychol Med. 1994 May; 24(2):385-95

42 Frith U, Happé F. Theory of Mind and SelfConsciousness: What Is It Like to Be Autistic? Mind Lang. 1999;14(1):82-9.

43 Nylander L, Lugnegård T, Hallerbäck MU. Autism spectrum disorders and schizophrenia spectrum disorders in adults: is there a connection? A literature review and some suggestions for future clinical research. Clin Neuropsychiatry J Treat Eval. 2008;5(1):43-54.

44 Hommer RE, Swedo SE. Schizophrenia and autism-related disorders. Schizophr Bull. 2015 Mar;41(2):313-4.

45 Høffding S, Martiny K. Framing a phenomenological interview: what, why and how. Phenomenol Cogn Sci. 2016;15(4):539-64.

46 Jansson L, Nordgaard J. The Psychiatric Interview for Differential Diagnosis. 1st ed. Basel: Springer International Publishing; 2016.

47 Parnas J. A disappearing heritage: the clinical core of schizophrenia. Schizophr Bull. 2011 Nov;37(6):1121-30.

48 Parnas J. Differential diagnosis and current polythetic classification. World Psychiatry. 2015 Oct;14(3):284-7.
49 Stanghellini G. The grammar of the psychiatric interview. A plea for the second-person mode of understanding. Psychopathology. 2007;40(2):69-74.

50 Nordgaard Frederiksen JE, Henriksen MG. Phenomenological Psychopathology and Quantitative Research. In: Stanghellini G, Raballo A, Broome M, Fernandez AV, Fusar-Poli P, Rosfort R, editors. The Oxford Handbook of Phenomenological Psychopathology. Oxford: University Press; 2018.

51 Zukauskas PR, Silton N, Assumpção FB Jr. Temporality and Asperger's syndrome. J Phenomenological Psychol. 2009;40(1):85-106.

52 Stanghellini G, Aragona M, editors. An Experiential Approach to Psychopathology. What Is It Like to Suffer from Mental Disorders? 1st ed. Basel: Springer International Publishing; 2016.

53 Elmose M. A conceptual framework for understanding characteristics of self-awareness associated with autism spectrum disorder. Scand J Child Adolesc Psychiatry Psychol. 2016;4(3):109-14.

54 Parnas J, Henriksen MG. Disordered self in the schizophrenia spectrum: a clinical and research perspective. Harv Rev Psychiatry. 2014 Sep-Oct;22(5):251-65.

55 Bizzari V. A Phenomenological Approach to Psychopathologies: an Embodied Proposal. InterCultural Philos J. 2018;2018(1):13256.

56 Sass L, Pienkos E, Skodlar B, Stanghellini G, Fuchs T, Parnas J, et al. EAWE. Psychopathology. 2017;50(1):10-54.

57 Binswanger L, editor. Grundformen und Erkenntnis menschlichen Daseins. 3rd ed. Zürich: Ernst Reinhardt Verlag; 1962.

58 Minkowski E, editor. Lived Time: Phenomenological and Psychopathological Studies. 1st ed. Evanston: Northwestern University Press; 1970.

59 Urfer A. Phenomenology and Psychopathology of Schizophrenia: The Views of Eugene Minkowski. In: Philosophy, Psychiatry, \&. Psychology. Baltimore (MD): Johns Hopkins University Press; 2001. pp. 279-89.

60 Trevarthen C, Hubley P. Secondary intersubjectivity: Confidence, confiding and acts of meaning in the first year. In: Lock A, editor. Action, Gesture and Symbol: The Emergence of Language. London: Academic Press; 1978.

61 Trevarthen C. Communication and cooperation in early infancy: a description of primary intersubjectivity. In: Bullowa M, editor. Before Speech: The Beginning of Interpersonal Communication. Cambridge: Cambridge University Press; 1979. pp. 321-72.

62 Jaspers K, editor. General Psychopathology. Volume 1. Baltimore (MD): Johns Hopkins University Press; 1997.

63 Merleau-Ponty M. Phenomenology of Perception. Abingdon: Routledge; 2014. 